\title{
General Psychiatry Study of brain morphology change in Alzheimer's disease and amnestic mild cognitive impairment compared with normal controls
}

\author{
Huanqing Yang, ${ }^{1,2}$ Hua Xu, ${ }^{1,2}$ Qingfeng $\mathrm{Li}^{3}{ }^{3}$ Yan Jin, ${ }^{4}$ Weixiong Jiang, ${ }^{5}$ \\ Jinghua Wang, ${ }^{1,2}$ Yina Wu, ${ }^{1,2}$ Wei Li, ${ }^{1,2}$ Cece Yang, ${ }^{1,2}$ Xia Li, ${ }^{1,2}$ Shifu Xiao, ${ }^{1,2}$ \\ Feng Shi, ${ }^{3}$ Tao Wang ${ }^{1,2}$
}

To cite: Yang H, Xu H, Li Q, et al. Study of brain morphology change in Alzheimer's disease and amnestic mild cognitive impairment compared with normal controls. General Psychiatry 2019;32:e100005. doi:10.1136/ gpsych-2018-100005

HY, HX and QL contributed equally.

HY, HX and QL are joint first authors.

Received 15 August 2018 Revised 13 March 2019 Accepted 15 March 2019

\section{Check for updates}

(c) Author(s) (or their employer(s)) 2019. Re-use permitted under CC BY-NC. No commercial re-use. See rights and permissions. Published by BMJ.

For numbered affiliations see end of article.

Correspondence to Dr Tao Wang: wtshhwy@163.com

Dr Feng Shi; feng.shi@united-imaging.com

\section{ABSTRACT}

Background With an aggravated social ageing level, the number of patients with Alzheimer's disease (AD) is gradually increasing, and mild cognitive impairment (MCl) is considered to be an early form of Alzheimer's disease. How to distinguish diseases in the early stage for the purposes of early diagnosis and treatment is an important topic.

Aims The purpose of our study was to investigate the differences in brain cortical thickness and surface area among elderly patients with $\mathrm{AD}$, elderly patients with amnestic $\mathrm{MCl}$ (aMCl) and normal controls (NC).

Methods $20 \mathrm{AD}$ patients, $21 \mathrm{aMCls}$ and $25 \mathrm{NC}$ were recruited in the study. FreeSurfer software was used to calculate cortical thickness and surface area among groups.

Results The patients with AD had less cortical thickness both in the left and right hemisphere in 17 of the 36 brain regions examined than the patients with $\mathrm{aMCl}$ or $\mathrm{NC}$. The patients with $\mathrm{AD}$ also had smaller cerebral surface area both in the left and right hemisphere in 3 of the 36 brain regions examined than the patients with $\mathrm{aMCl}$ or NC. Compared with the NC, the patients with aMCl only had slight atrophy in the inferior parietal lobe of the left hemisphere, and no significant difference was found. Conclusion $\mathrm{AD}$, as well as $\mathrm{aMCl}$ (to a lesser extent), is associated with reduced cortical thickness and surface area in a few brain regions associated with cognitive impairment. These results suggest that cortical thickness and surface area could be used for early detection of $A D$.

\section{INTRODUCTION}

Alzheimer's disease (AD), the most common form of dementia, accounts for $50 \%-60 \%$ of all cases. It is a progressive, irreversible and currently incurable neurodegenerative disease. ${ }^{2}$ The main clinical manifestations are continuous degeneration of cognitive function, comprehension, judgement, memory and self-control, even total loss occurs. Dramatic loss of cortical neurons in $\mathrm{AD}$ results in severe atrophy of the cerebral grey matter, causing progressive decline in cognition and brain function. Mild cognitive impairment (MCI), a potential precursor to or early form of $\mathrm{AD}$, has the same aetiology, but to a lesser degree. ${ }^{3}$ Approximately 10 to $15 \%$ of patients with MCI experience progression to $\mathrm{AD} .{ }^{45}$ Amnestic MCI (aMCI), a subtype of MCI, is characterised by primary memory deficits, and patients with aMCI have a high risk of progression to $\mathrm{AD}{ }^{6}$

In the current study, we compared the surface-based measures of cerebral cortical thickness and surface area among patients with $\mathrm{AD}$, patients with aMCI and normal elderly controls. The brain is composed of grey matter and white matter. Cortical thickness is defined as the distance from the interface between cerebrospinal fluid and grey matter to the interface between the white matter cortex and the grey matter cortex. ${ }^{7}$ Compared with normal elderly individuals, elderly patients with MCI have been shown to have significantly reduced cortical thickness, mainly in the hippocampus, parahippocampal gyrus, temporal lobe, fusiform gyrus, posterior cingulate, and other areas, and these abnormalities are even more pronounced in elderly patients with $\mathrm{AD} .{ }^{8-10}$ Surface area is the average area of the triangles that touch the vertex on the pial surface. ${ }^{11}$ The complexity of the folding of the sulci, which are numerous in the cerebral cortex, affects the size of the surface area.

Although previous studies have uncovered abnormal development of brain structures in $\mathrm{AD}$, results pertaining to the relationship between memory deficits and grey matter volume in patients with $\mathrm{AD}$ and MCI have not been consistent. ${ }^{12}$ On the basis of previous findings of widespread abnormalities in brain structure in $\mathrm{AD}$, we expected to find significant alterations in cortical thickness and surface area in patients with $\mathrm{AD}$ and aMCI. 


\section{MATERIALS AND METHODS}

\section{Participants}

All volunteers and their legal guardians involved gave informed consent. Eligible patients recruited here are patients with $\mathrm{AD}$ who met the criteria of dementia according to the Diagnostic and Statistical Manual of Mental Disorders, and aMCI was diagnosed based on the previously published criteria. ${ }^{12}$ Age-matched and sex-matched normal control (NC) subjects were also enrolled. Before enrolment, patients were asked to provide their medical history and perform physical and neurological examinations, laboratory tests and T1-weighted MRI scans. Patients with AD whose course of disease was more than 2 years without treatment and patients with aMCI without treatment in the disease course for more than 1 year were chosen. Patients with any of the following were excluded: a neurological disorder, any physical illness (such as hepatitis, brain tumour, trauma or epilepsy) or a history of major depression, bipolar disorder, schizophrenia and substance abuse or dependence in the previous 180 days. The control subjects did not have any cognitive complaints or functional impairments. ${ }^{13}$

In order to accommodate the low education level of the elder Chinese, we amended the aMCI diagnostic criteria of the Petersen Mini Mental States Examination $(\text { MMSE })^{14}$ and evaluated the cognitive status of each participant. The scores $<24$ indicated $\mathrm{AD}$, scores ranging from 24 to 26 indicated aMCI and scores $>26$ were considered normal. All scores were consistent with the patient's diagnosis.

\section{MR image acquisition}

MRI was used for scanning participants using a Siemens MAGNETOM VERIO 3T scanner (Munich) at Shanghai Mental Health Center. T1-weighted images were obtained with 128 sagittal slices using the three-dimensional magnetisation-prepared rapid acquisition gradient echo sequence with the following parameters: $T R=2530 \mathrm{~ms}$, $\mathrm{TE}=3.39 \mathrm{~ms}$, flip angle $=7^{\circ}$ and spatial resolution $=1 \times 1 \mathrm{x}$ $1.3 \mathrm{~mm}^{3}$, and the acquisition time was $8 \min 7 \mathrm{~s}$. Images were reviewed and discarded, if there were any pathological findings.

\section{Image processing}

All MRI studies were processed on the same workstation using FreeSurfer V.5.3. Cortical surface reconstruction was performed to calculate cortical thickness and surface area at each vertex. ${ }^{15}$ The Automated Anatomical Labelling template is a widely used high-resolution T1-weighted brain parcellation based on a single adult subject. ${ }^{16}$ FreeSurfer automatically parcelled the cortex into 36 gyral-based regions of interest (ROIs) per hemisphere (figure 1) using several processing steps, including skull stripping, spatial transformation, atlas registration, surface reconstruction, spherical surface mapping and atlas-based regional parcellation. Both grey matter/white matter and grey matter/cerebrospinal fluid surfaces were reconstructed using spatial intensity gradients across

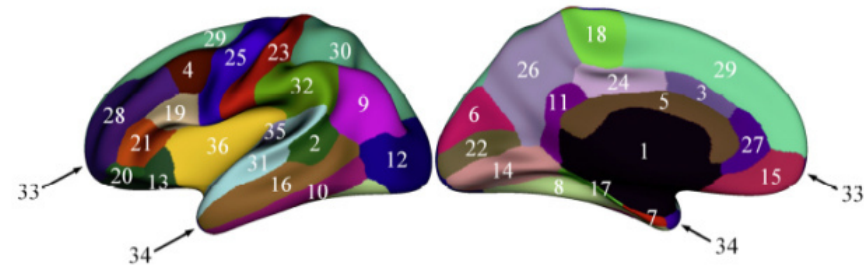

\begin{tabular}{|l|l|}
\hline 1. Unlabelled Subcortical Region & 2. Bank of the Superior Temporal Sulcus \\
\hline 3. Caudal Anterior-cingulate Cortex & 4. Caudal Middle Frontal Gyrus \\
\hline 5. Unmeasured Corpus Callosum & 6. Cuneus Cortex \\
\hline 7. Entorhinal Cortex & 8. Fusiform Gyrus \\
\hline 9. Inferior Parietal Cortex & 10. Inferior Temporal Gyrus \\
\hline 11. Isthmus-cingulate Cortex & 12. Lateral occipital cortex \\
\hline 13. Lateral orbital frontal cortex & 14. Lingual gyrus \\
\hline 15. Medial orbital frontal cortex & 16. Middle temporal gyrus \\
\hline 17. Parahippocampal gyrus & 18. Paracentral lobule \\
\hline 19. Pars opercularis & 20. Pars orbitalis \\
\hline 21. Pars triangularis & 22. Pericalcarine cortex \\
\hline 23. Postcentral gyrus & 24. Posterior-cingulate cortex \\
\hline 25. Precentral gyrus & 26. Precuneus cortex \\
\hline 27. Rostral anterior cingulate cortex & 28. Rostral middle frontal gyrus \\
\hline 29. Superior frontal gyrus & 30. Superior parietal cortex \\
\hline 31. Superior temporal gyrus & 32. Supramarginal gyrus \\
\hline 33. Frontal pole & 34. Temporal pole \\
\hline 35. Transverse temporal cortex & 36. Insula cortex \\
\hline
\end{tabular}

Figure 1 Regions of interest in the left hemisphere.

tissue classes. The cerebral cortex was then parcelled into regions on the basis of the folding structure of gyri and sulci. ${ }^{16}$ Cortical thickness was calculated as the shortest distance between the grey matter/white matter surface and the pial surface. ${ }^{17}$ More technical details of these procedures were described previously. ${ }^{15} 18$ The results of the automated segmentation, surface reconstruction and parcellation process were manually inspected for all participants.

\section{Statistical analyses}

Cortical thickness and surface area maps were smoothed using a $20 \mathrm{~mm}$ Gaussian kernel. ${ }^{19}$ Then, variance analysis was applied to the measures of cortical thickness and surface area to determine whether there were significant differences in brain structure between the patients with $\mathrm{AD}$, aMCI and the control subjects. We used a false discovery rate of 0.05 , corrected for multiple comparisons at the vertex level. We also modelled age and the whole brain volume as covariates of no interest to minimise any confounding effects of these variables.

\section{RESULTS}

\section{Patient characteristics}

The figure 2 shows the flow diagram of the study. We recruited a total of 20 patients with $\mathrm{AD}, 21$ patients with aMCI and 25 age-matched and sex-matched NC. The mean (SD) age for the entire cohort was 71.74 (7.373) 


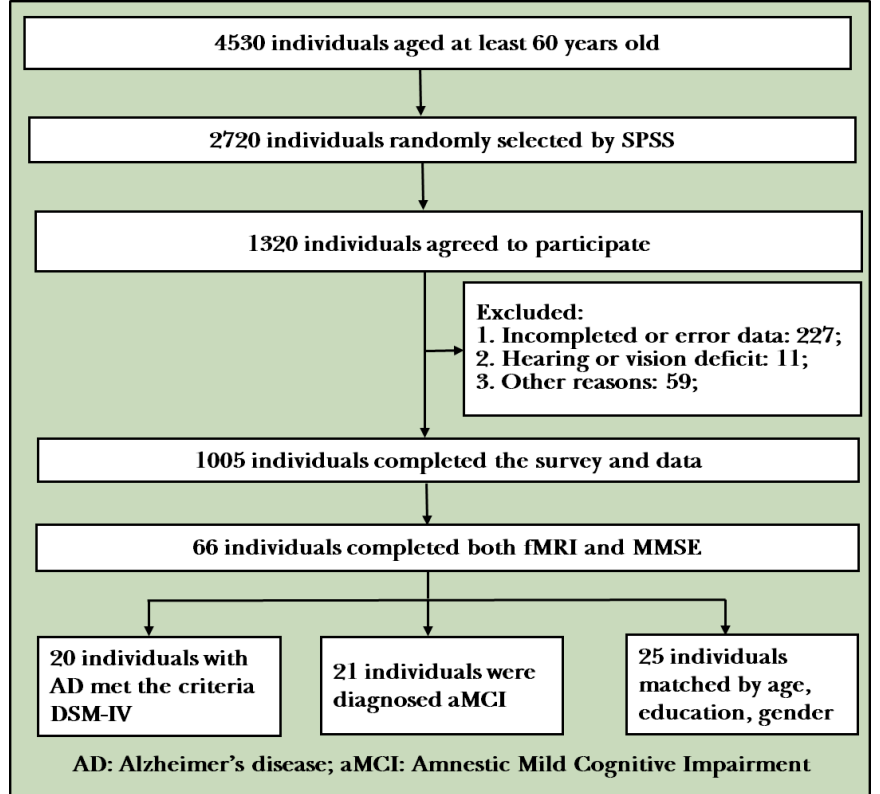

Figure 2 Flowchart of the study

years and the mean education level (measured by years completed) was 11.19 (2.83) years. Demographic and clinical data for participants by group are presented in table 1 .

\section{Cortical thickness}

There was no marked difference of cortical thickness in both left and right hemisphere between patients with aMCI and NC (figure 3A-D).

In the left hemisphere, cortical thickness was significantly reduced in patients with $\mathrm{AD}$ compared with $\mathrm{NC}$ in 22 areas of the cerebral cortex, including lateral occipital cortex, lingual gyrus, superior parietal cortex, precentral gyrus, inferior parietal cortex, isthmus cingulate cortex, postcentral gyrus, cuneus cortex, pericalcarine cortex, insula cortex, precuneus cortex, supramarginal gyrus, paracentral lobule, pars triangularis, posterior-cingulate cortex, transverse temporal cortex, fusiform gyrus, rostral anterior cingulate cortex, caudal anterior-cingulate

Table 1 Demographic and clinical features of patients with $\mathrm{AD}$, patients with $\mathrm{aMCl}$ and normal controls.

\begin{tabular}{llll}
\hline Variable & AD & aMCl & Control \\
\hline Participants (n) & 20 & 21 & 25 \\
$\begin{array}{l}\text { Sex (male/ } \\
\text { female) }\end{array}$ & $7 / 13$ & $12 / 9$ & $8 / 17$ \\
$\begin{array}{l}\text { Mean age (SD) } \\
\text { (years) }\end{array}$ & $70.8(5.63)$ & $69.6(7.64)$ & $73.56(7.16)$ \\
$\begin{array}{l}\text { Mean level of } \\
\text { education (SD) } \\
\text { (years) }\end{array}$ & $11.45(3.44)$ & $10.95(2.51)$ & $10.56(2.33)$ \\
$\begin{array}{l}\text { Mean MMSE } \\
\text { score (SD) }\end{array}$ & $16.50(5.62)$ & $26.24(1.77)$ & $28.60(1.13)$ \\
\hline
\end{tabular}

$A D$, Alzheimer's disease; MMSE, mini-mental status examination; aMCl, amnestic mild cognitive impairment.

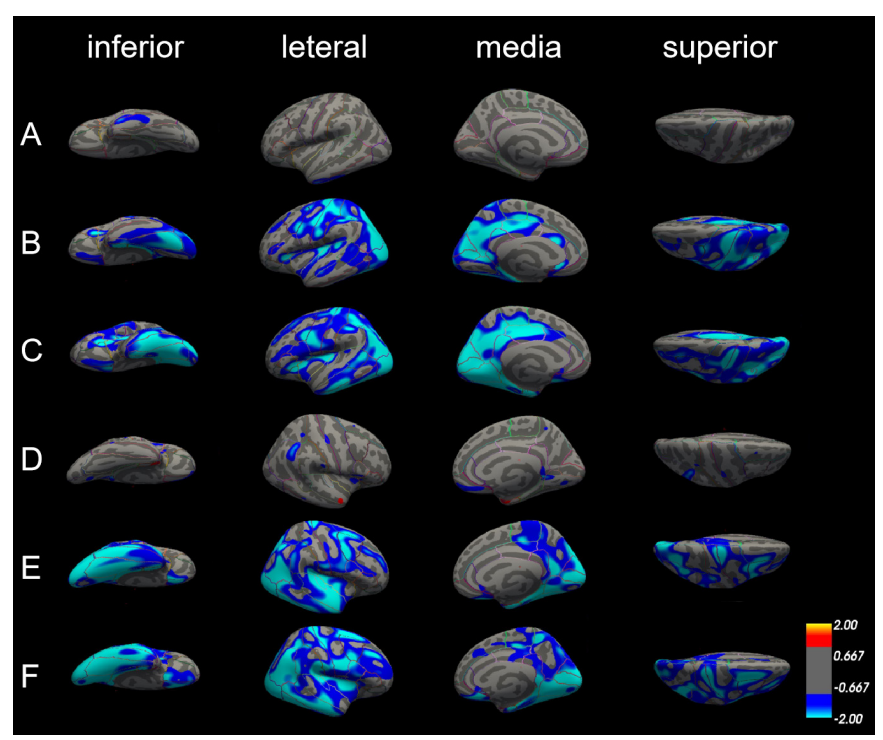

Figure 3 Differences in cortical thickness among patients with Alzheimer's disease (AD), patients with amnestic mild cognitive impairment (aMCl) and normal controls (NC). The comparison is made from four directions (inferior, lateral, medial and superior) in the left hemisphere ( $A, B$ and $C)$ and right hemisphere (D, E and F), respectively. (A) and (D) Cortical thickness did not significantly differ between patients with aMCl and $\mathrm{NC}$ in both left and right hemisphere. (B) and

(E) The blue areas showed significantly reduced cortical thickness in patients with aMCl compared with NC. (C) and (F) The blue areas showed significantly reduced cortical thickness in patients with $\mathrm{AD}$ compared with aMCl.

cortex, superior temporal gyrus, superior frontal gyrus and caudal middle frontal gyrus. In the right hemisphere, there is no significant difference in cortical thickness in caudal anterior-cingulate cortex, isthmus cingulate, pars triangularis, rostral anterior cingulate cortex, transverse temporal cortex, but in the entorhinal cortex, medial orbitofrontal cortex, middle temporal gyrus, parahippocampal gyrus, the cortical thickness is reduced significantly (figure 3B,E, table 2).

Compared with patients with aMCI, cortical thickness was also significantly reduced in patients with $\mathrm{AD}$; the thinner areas were similar as those between patients with $\mathrm{AD}$ and NC. The different areas were mainly in the left hemisphere, including pericalcarine cortex, inferior parietal cortex, inferior temporal gyrus, cuneus cortex, precuneus cortex, posterior-cingulate cortex, lateral occipital cortex, postcentral gyrus, insula cortex, parahippocampal gyrus, entorhinal cortex, isthmus-cingulate cortex, lateral orbitofrontal cortex, fusiform gyrus, superior parietal cortex, middle temporal gyrus, pars triangularis, precentral gyrus, paracentral lobule, caudal middle frontal gyrus, and lingual gyrus. But in the areas such as lateral orbitofrontal cortex, paracentral lobule, pars triangularis, posterior-cingulate cortex and superior parietal cortex only can find reduce in left side, the areas such as medial orbital frontal cortex, superior frontal gyrus and supramarginal gyrus show significantly reduce in the right side (figure 3C,F, table 3). 


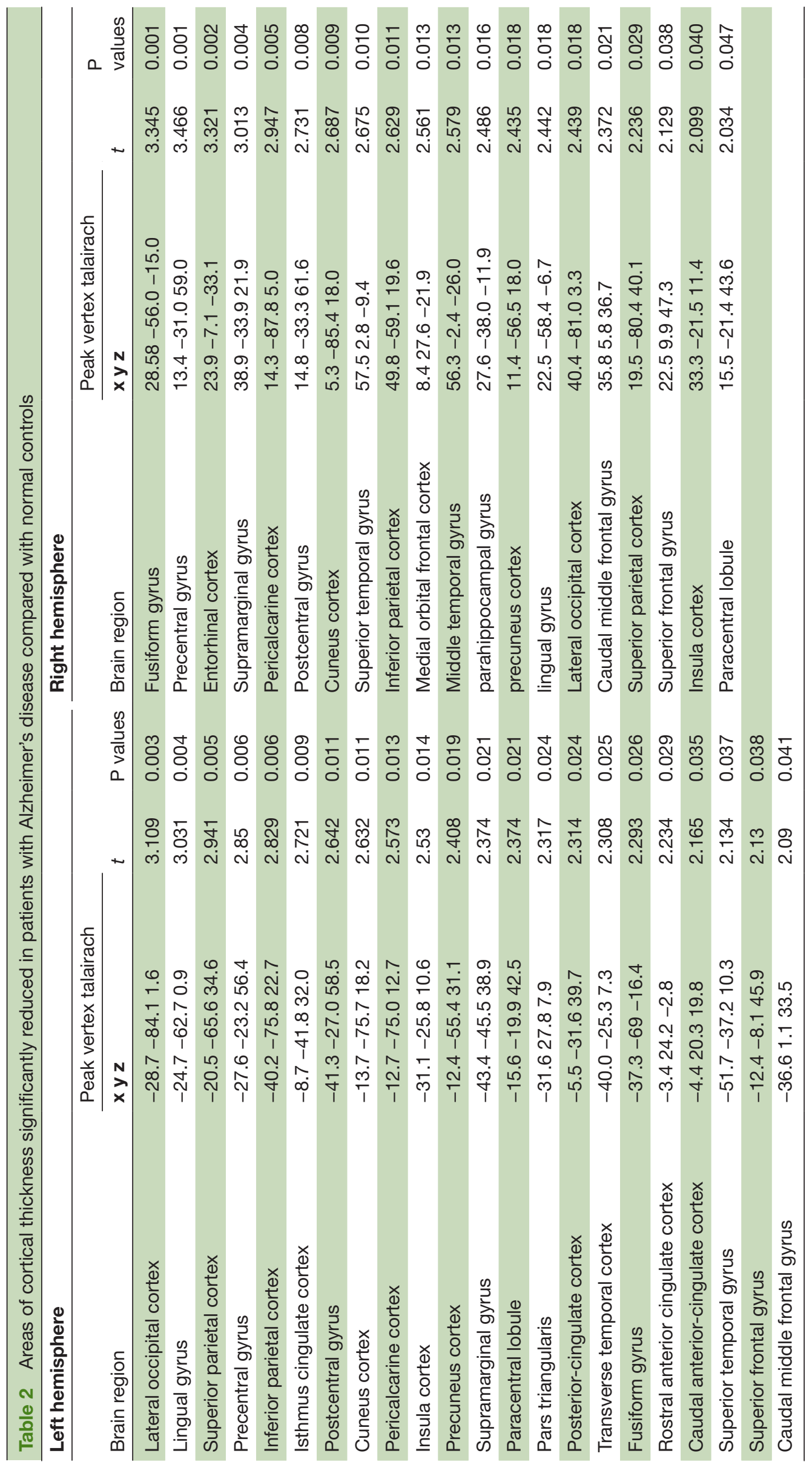




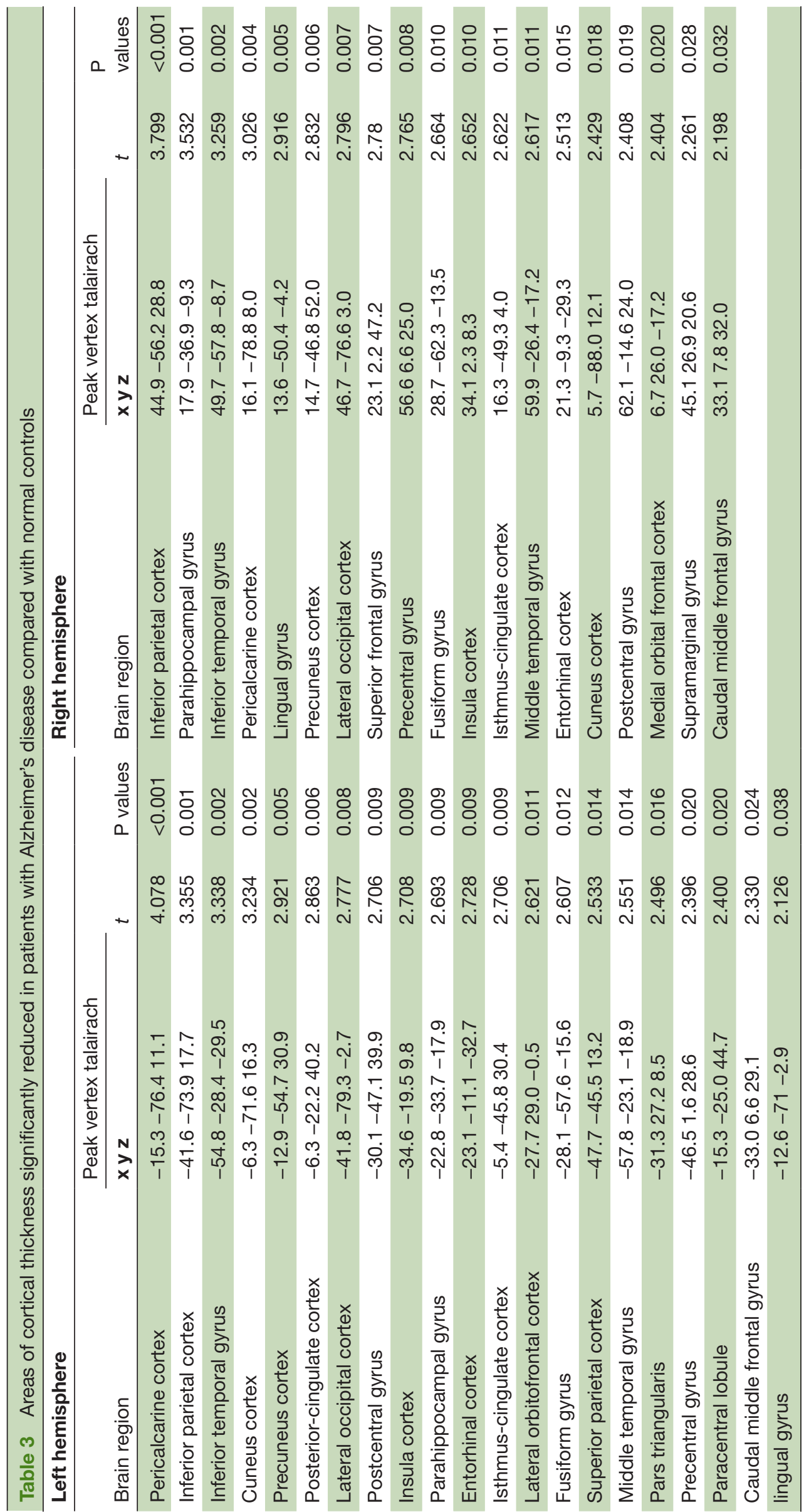




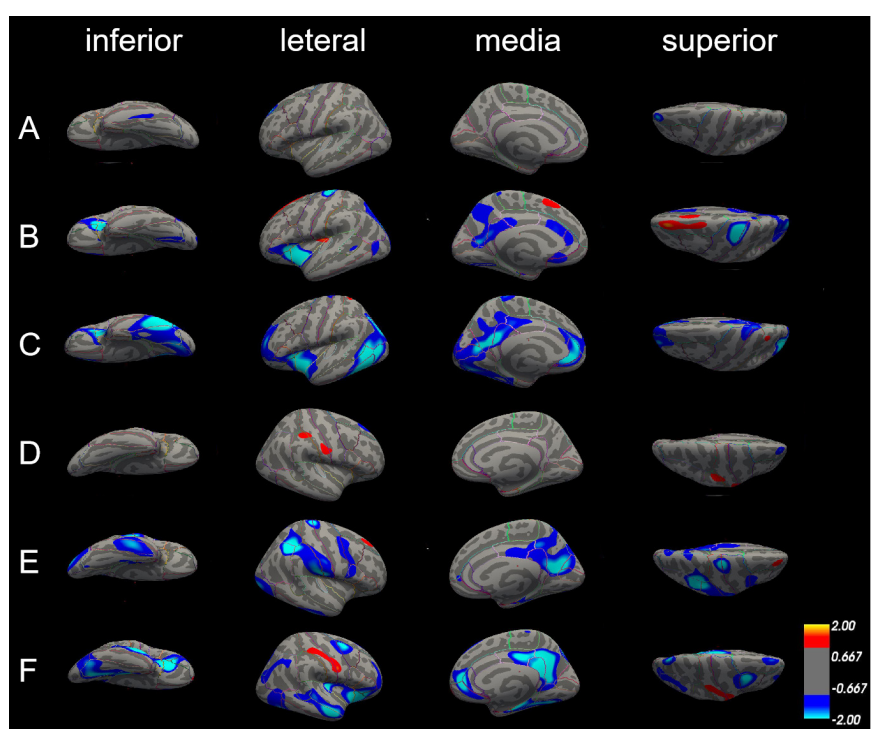

Figure 4 Differences in surface area among patients with Alzheimer's disease (AD), patients with amnestic mild cognitive impairment (aMCl) and normal controls (NC). The comparison is made from four directions (inferior, lateral, medial and superior) in the left hemisphere (A, B and $C$ ) and right hemisphere (D, E and F), respectively. (A) and (D) Surface area did not significantly differ between patients with $\mathrm{aMCl}$ and $\mathrm{NC}$ in both left and right hemisphere. (B) and (E) The blue areas showed significantly reduced surface area in patients with aMCl compared with NC. (C) and (F) The blue areas showed significantly reduced surface area in patients with $\mathrm{AD}$ compared with aMCl.

\section{Surface area}

There were no significant differences in the surface area in the left hemisphere between patients with aMCI and $\mathrm{NC}$; only some differences in the lateral occipital cortex of the right hemisphere (figure 4A,D).

Compared with NC, patients with AD exhibited several significantly smaller cerebral surface areas. Only precentral gyrus and superior parietal cortex were reduced in both left and right hemisphere; surface areas of insula cortex, lateral orbitofrontal cortex and pars triangularis were only reduced in the left hemisphere, and surface areas of cuneus cortex, inferior parietal cortex, postcentral gyrus, precentral gyrus, and supramarginal gyrus were only reduced in the right hemisphere (figure 4B,E, table 4).

Differences in the surface area between patients with $\mathrm{AD}$ and with aMCI in the left and right hemisphere were also similar. The left and right hemispheres have several areas of common reduction, such as fusiform gyrus, insula cortex, isthmus-cingulate cortex, middle temporal gyrus, precuneus cortex, superior parietal cortex, and they also have smaller surface areas that belong to their own parts. The surface area of inferior parietal cortex, inferior temporal gyrus, lateral occipital cortex, pars triangularis, pericalcarine cortex and rostral anterior cingulate cortex was significantly reduced in the left hemisphere; however, the surface area of caudal middle frontal gyrus, lateral orbitofrontal cortex, medial orbital frontal cortex, parahippocampal gyrus, posterior-cingulate cortex and

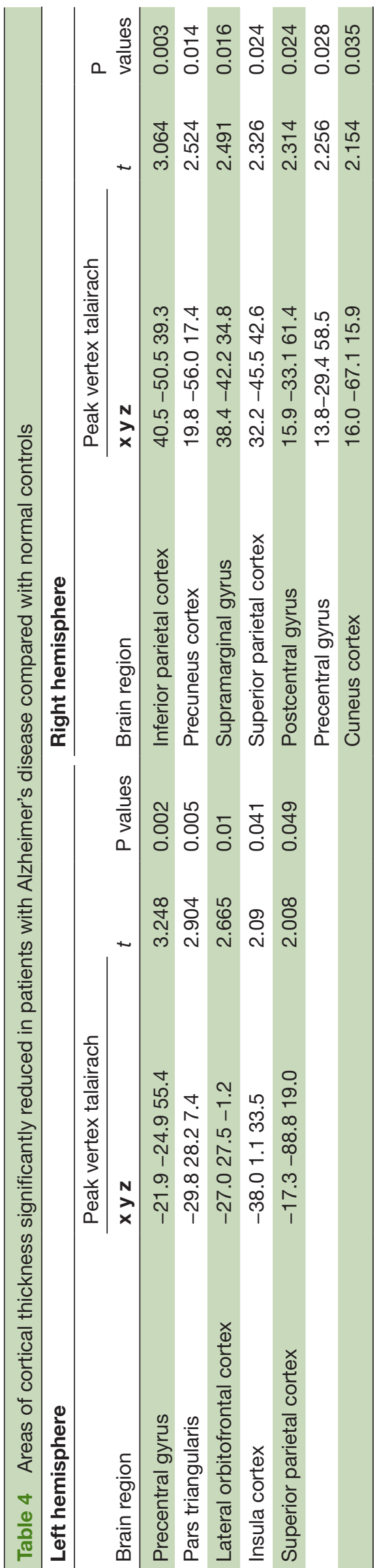


superior frontal gyrus was reduced in the right side (figure 4C,F, table 5).

\section{DISCUSSION}

\section{Main findings}

In the current study, the main goal was to investigate changes in cortical anatomy, specifically cortical thickness and surface area, in patients with $\mathrm{AD}$ and aMCI compared with NC. As predicted, compared with patients with aMCI and NC, patients with $\mathrm{AD}$ had significant cortical thickness and surface area atrophy in a wide regions of the frontal, temporal and occipital lobes of the brain. In addition, we found that patients with aMCI showed atrophy between normal ageing and $\mathrm{AD}$. As aMCI is considered to be the prodromal stage of $\mathrm{AD}$, even in the preclinical phase, our results collectively indicate that the atrophy of cortical thickness and surface area in aMCI begins in the temporal lobe, but the range of atrophy gradually expands as the disease progresses.

As can be found from our results, the reduction in cortical thickness is mainly concentrated in pericalcarine cortex, supramarginal gyrus, cuneus cortex, lateral occipital cortex, precuneus cortex, paracentral lobule, fusiform gyrus, superior frontal gyrus, lateral occipital cortex, entorhinal cortex, inferior parietal cortex, isthmus-cingulate cortex, postcentral gyrus, superior parietal cotex, caudal middle frontal gyrus, insula cortex and precentral gyrus. We found the pars triangularis was the only region that showed significant reduction in cortical thickness in the left hemisphere. The pars triangularis is part of the inferior frontal gyrus and is also known as Brodmann area (BA) 45. Together with BA44, BA45 comprises Broca's area, a region with functions linked to speech production, such as semantic decision tasks and generation tasks. In the linguistic information processing, it has been proven that there is a left hemisphere dominant in Broca area. ${ }^{20}$ The lesions of BA45 cause the characteristic manifestations of aphasia in left hemisphere dominant individuals. The vast majority of right-handed adults often suffer from cerebral cortex injuries on the left side of the brain. The BA44 area lesion in the right hemisphere did not show significant impairment of language activity. However, the injury to the Broca area of the left hemisphere resulted in severe aphasia. Brain lateralisation refers to the structural and functional asymmetry of the left and right hemispheres of the brain. Usually, the language function shows an obvious left hemisphere dominance, ${ }^{21}$ while cognitive control shows the right hemisphere dominance. ${ }^{22}$ Liu and colleagues investigated the topological properties of the whole brain networks by using functional MRI in patients with $\mathrm{AD}$, patients with MCI and age-matched healthy subjects. Compared with NC, patients with MCI showed decreased nodal centrality mainly in the right pars triangularis, right superior parietal cortex and left hippocampus. ${ }^{23}$ The result in our study showed that compared with NC, aMCI cortical thickness was not significantly reduced at the pars triangularis, whereas the cortex thickness of the left pars triangularis was significantly

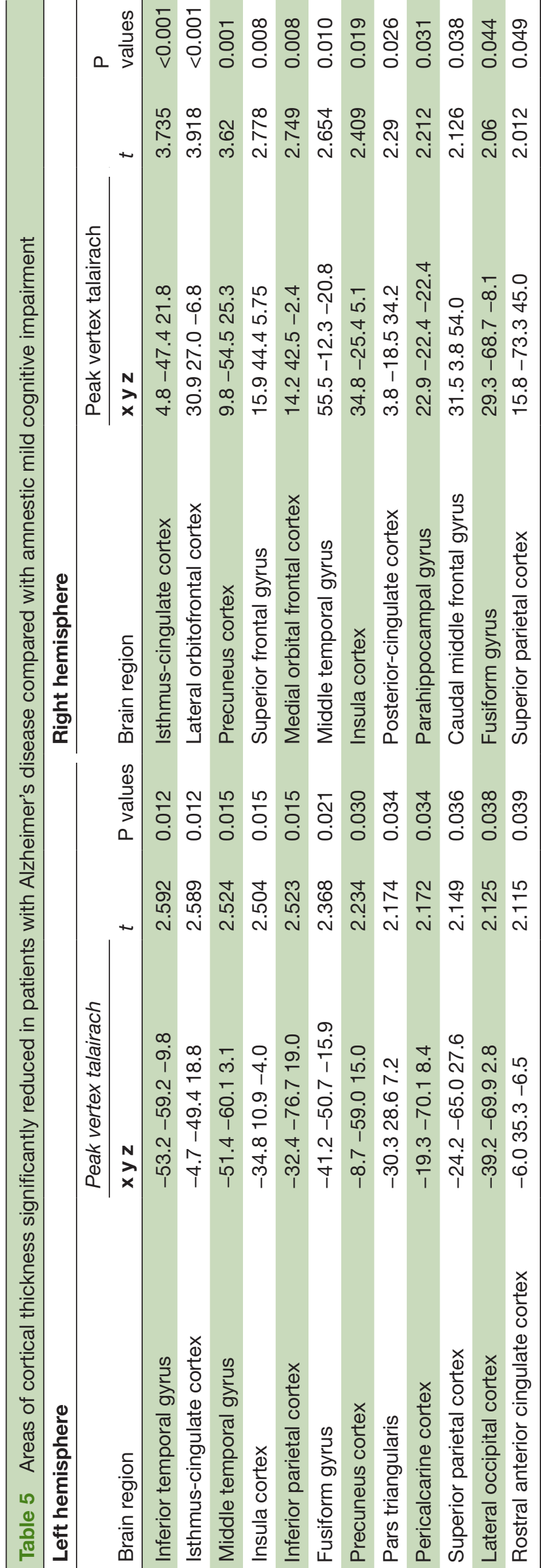


atrophied in AD compared with aMCI and NC. Consider that language production has not been significantly affected in the early stages of cognitive decline. With the gradual decline of cognitive function, the language production function begins to be significantly impaired, which is consistent with the clinical performance of patients with $\mathrm{MCI}$ and $\mathrm{AD}$. In addition, medial orbital frontal cortex and middle temporal gyrus showed that significant cortex thickness reduction only existed on the right hemisphere. The most important area of the brain related to memory is the medial temporal lobe, including the hippocampus and entorhinal cortex. $^{24}$

Another result in our study was that compared with aMCI and NC, respectively, the precuneus cortex, insula cortex and superior parietal cotex of AD showed significant difference in surface area. The precuneus cortex is a part of the superior parietal lobule on the medial surface, forward of the cuneus. It contains three subdivisions: the sensorimotor anterior region, cognitive/ associative central region and visual posterior region. It participates in various processes such as episodic memory, visual space processing, self-reflection and consciousness. ${ }^{25}$ The forward part of the precuneus is related to the mental imagery concerning the self, and the posterior areas are involved with episodic memory. ${ }^{26}$ It is associated with the left prefrontal cortex and involved with the recall of episodic memory, including past self-related episodes. ${ }^{27} 28$ In addition, we found that consistent with the reduction in cortical thickness, the pars triangularis of the patients with $\mathrm{AD}$ also had a significant reduction in cortical surface area in the left cerebral hemisphere, with the medial orbital frontal cortex and middle temporal gyrus decreasing in the right cerebral hemisphere.

Several studies have suggested that the grey matter atrophy in $\mathrm{AD}$ is asymmetric between the left and right hemispheres and that it is more serious on the left side than on the right. However, this asymmetry is controversial. ${ }^{29}{ }^{30}$ Another study found that atrophy of the left and right hemispheres of the cerebral cortex in patients with mild $\mathrm{AD}$ presented to be basically symmetrical, but the atrophy of the left posterior parietal lobe and dorsolateral cortex was more severe than that of the right side. ${ }^{8}$ Our results were similar to those, compared with the NC group, the cortical thickness and surface area in the right hemisphere of patients with aMCI had no significant difference; only a slight atrophy in the lateral parietal lobe of the left hemisphere, but did not reach significant difference. The surface area and cortical thickness of the left hemisphere of the patients with $\mathrm{AD}$ were significantly lower than the patients with aMCI. Although the left and right sides of $\mathrm{AD}$ had slight differences in atrophy, the atrophy aeras were similar. Therefore, we can conclude that the asymmetry of the atrophy between the left and right hemisphere becomes less pronounced with the development of $\mathrm{AD}$, and the atrophy diffused into the whole brain.

As mentioned above, our study showed that patients with $\mathrm{AD}$ have been shown to have areas of atrophy throughout the brain, and the atrophy is concentrated in areas related to learning, memory, language understanding, information integration and other cognitive functions. ${ }^{31}$ Our study also showed that both cortical thickness and surface area were markedly reduced in the prefrontal lobe and lateral occipital cortex in patients with $\mathrm{AD}$, suggesting that these are consistent areas of atrophy in $\mathrm{AD}$. Our results also suggest that cortical thickness and surface area can detect how the brain changes during the process of cognitive ageing. Atrophy in MCI subjects was spatially more restricted than in AD but occurred at similar locations. Thus, our findings suggest that cortical thickness and surface area may be a sensitive measure for characterising grey matter atrophy in the early stages of $\mathrm{AD}$, which may help with early detection of the disease.

\section{Limitations}

First, the sample size was not big enough; we will increase the number of patients analysed in follow-up studies so that more differences can be examined among the groups. Second, traditional measurement methods of ROI have some limitations. The choice of ROI is based on the researchers' existing experience, and the positioning of ROI is somewhat subjective among researchers. Therefore, the specific ROI should be reviewed in any comparisons to other studies. Finally, the current study was based on findings from only one MRI study per patient at a specific stage of the disease. Although our use of multiple patient groups allowed for general comparison over the course of disease progression, further research should take a more longitudinal approach to gain more insight into how structural changes relate to cognitive status over time within the same patients. In future studies, we also plan to further subdivide patients with $\mathrm{AD}$ on the basis of cognitive status. We will explore this topic further in the future.

\section{Implications}

In summary, we performed a systematic study on cortical thickness and surface area among the three groups: the $\mathrm{AD}$ group, the aMCI group and the NC group. Therefore, we found that the reduction of cortical thickness and surface area are associated with the development of $\mathrm{AD}$, and could be used for early detection of $\mathrm{AD}$.

\section{Author affiliations}

${ }^{1}$ Department of Geriatric Psychiatry, Shanghai Mental Health Center, Shanghai, China

${ }^{2}$ Alzheimer's Disease and Related Disorders Center, Shanghai Jiao Tong University, Shanghai, China

${ }^{3}$ Shanghai United Imaging Intelligence Co., Ltd, Shanghai, China

${ }^{4}$ IDEA Lab, Department of Radiology and BRIC, University of North Carolina at Chapel Hill, Chapel Hill, NC, United States

${ }^{5}$ Informational Science and Engineering Department, Hunan First Normal University, Changsha, China

Correction notice This article has been corrected since it was published Online First. This article was originally published without the correct Open Access licence.

Contributors HY and HX drafted the paper. HY and QL made the statistical analysis. YJ, WJ, JW, YW, WL and CY carried out the data collection. XL doublechecked the data. SX designed and was responsible for the study. TW carried out the research, while TW and FS supervised the data collection, statistical analysis 
and modified the paper. HY, HX and QL contributed equally to this work as co-first authors.

Funding TW was supported by the Collaborative Innovation Center for Translational Medicine at Shanghai Jiao Tong University School of Medicine TM201728, National Nature Science Foundation of China 81571298, Shanghai health system excellent talent training program (excellent subject leader) project 2017BR054, Shanghai Municipal Education Commission-Gaofeng Clinical Medicine Grant Support 20172029, Shanghai Pujiang Program 17PJD038.

Competing interests None declared.

Patient consent for publication Obtained.

Ethics approval This study was approved by the Ethical Committee of Shanghai Mental Health Center.

Provenance and peer review Not commissioned; externally peer reviewed.

Data sharing statement № additional data are available.

Open access This is an open access article distributed in accordance with the Creative Commons Attribution Non Commercial (CC BY-NC 4.0) license, which permits others to distribute, remix, adapt, build upon this work non-commercially, and license their derivative works on different terms, provided the original work is properly cited, appropriate credit is given, any changes made indicated, and the use is non-commercial. See: http://creativecommons.org/ licenses/by-nc/4.0/.

\section{REFERENCES}

1. Blennow $\mathrm{K}$, Leon MJD, Zetterberg $\mathrm{H}$. Alzheimer's disease. Lacent 2006;368:387-403.

2. Weihao Z, Zhijun $\mathrm{Y}$, Bin $\mathrm{H}$, et al. Novel cortical thickness pattern for accurate detection of Alzheimer's disease. Journal of Alzheimer's Disease 2015;48:995-1008.

3. Vinters HV. Neuropathology of amnestic mild cognitive impairment. Arch Neurol 2006;63:645-6.

4. Serrano CM, Dillon C, Leis A, et al. Mild cognitive impairment: risk of dementia according to subtypes. Actas espa?olas de psiquiatría 2013;41.

5. Grundman M, Petersen RC, Ferris SH. Mild cognitive impairment can be distinguished from Alzheimer disease and normal aging for clinical trials. Arch Neurol 2004:61:59-66.

6. Li S, Yuan X, Pu F, et al. Abnormal changes of multidimensional surface features using multivariate pattern classification in amnestic mild cognitive impairment patients. J Neurosci 2014;34:10541-53.

7. Lerch JP, Evans AC. Cortical thickness analysis examined through power analysis and a population simulation. Neurolmage 2005;24:163-73.

8. Baron JC, Chételat $\mathrm{G}$, Desgranges $\mathrm{B}$, et al. In vivo mapping of gray matter loss with voxel-based morphometry in mild Alzheimer's disease. Neurolmage 2001;14:298-309.

9. Bell-McGinty S, Lopez OL, Meltzer CC, et al. Differential cortical atrophy in subgroups of mild cognitive impairment. Arch Neurol 2005;62:1393-7.

10. Chetelat G, Desgranges B, Landeau B, et al. Direct voxel-based comparison between grey matter hypometabolism and atrophy in Alzheimer's disease. Brain 2007;131:60-71.

11. Rakic P. Defects of neuronal migration and the pathogenesis of cortical malformations. Prog Brain Res 1988;73:15-37.
12. Ferreira LK, Diniz BS, Forlenza OV, et al. Neurostructural predictors of Alzheimer's disease: a meta-analysis of VBM studies. Neurobiology of Aging 2011;32:1733-41.

13. McKhann GM, Knopman DS, Chertkow $\mathrm{H}$, et al. The diagnosis of dementia due to Alzheimer's disease: recommendations from the National Institute on Aging-Alzheimer's association workgroups on diagnostic guidelines for Alzheimer's disease. Alzheimers Dement 2011;7:263-9.

14. Folstein MF, Folstein SE, McHugh PR, et al. A practical method for grading the cognitive state of patients for the clinician. $J$ Psychiatr Res 1975;12:189-98.

15. Fischl B, van der Kouwe A, Destrieux C. Automatically Parcellating the human cerebral cortex. Cereb Cortex 2004;14:11-22.

16. Tzourio-Mazoyer N, Landeau B, Papathanassiou D, et al. Automated anatomical labeling of activations in SPM using a macroscopic anatomical parcellation of the MNI MRI single-subject brain Neurolmage 2002;15:273-89.

17. Fischl B, Dale AM. Measuring the thickness of the human cerebral cortex from magnetic resonance images. Proceedings of the National Academy of Sciences 2000;97:11050-5.

18. Desikan RS, Ségonne F, Fischl B, et al. An automated labeling system for subdividing the human cerebral cortex on MRI scans into gyral based regions of interest. Neurolmage 2006;31:968-80.

19. Karama S, Bastin ME, Murray C, et al. Childhood cognitive ability accounts for associations between cognitive ability and brain cortical thickness in old age. Molecular Psychiatry 2014;19:555-9.

20. Bookheimer S. Functional MRI of language: new approaches to understanding the cortical organization of semantic processing. Annual Review of Neuroscience 2002;25:151-88.

21. Toga AW, Thompson PM. Mapping brain asymmetry. Nat Rev Neurosci 2003;4:37-48.

22. Smith DV, Clithero JA, Rorden C, et al. Decoding the anatomical network of spatial attention. Proc Natl Acad Sci USA 2013;110:1518-23.

23. Liu Z, Zhang $\mathrm{Y}$, Yan $\mathrm{H}$, et al. Altered topological patterns of brain networks in mild cognitive impairment and Alzheimer's disease: a resting-state fMRI study. Psychiatry Research: Neuroimaging 2012;202:118-25.

24. Buckner RL, Andrews-Hanna JR, Schacter DL. The brain's default network: anatomy, function, and relevance to disease. Ann N Y Acad Sci 2008;1124:1-38.

25. Margulies DS, Vincent JL, Kelly C, et al. Precuneus shares intrinsic functional architecture in humans and monkeys. Proceedings of the National Academy of Sciences 2009;106:20069-74.

26. Fletcher PC, Frith CD, Baker SC, et al. The mind's Eye-Precuneus activation in memory-related imagery. Neurolmage 1995;2:195-200.

27. Lundstrom B, Petersson KM, Andersson J. Isolating the retrieval of imagined pictures during episodic memory: activation of the left precuneus and left prefrontal cortex. Neurolmage 2003;20:1934-43.

28. Sadigh-Eteghad S, Majdi A, Farhoudi M, et al. Different patterns of brain activation in normal aging and Alzheimer's disease from cognitional sight: meta analysis using activation likelihood estimation. J Neurol Sci 2014;343:159-66.

29. Thompson PM, Hayashi KM, de Zubicaray G, et al. Dynamics of gray matter loss in Alzheimer's disease. The Journal of Neuroscience 2003;23:994-1005.

30. Karas G, Sluimer J, Goekoop R, et al. Amnestic mild cognitive impairment: structural MR imaging findings predictive of conversion to Alzheimer disease. AJNR Am J Neuroradiol 2008;29:944-9.

31. Edmonds EC, Eppig J, Bondi MW, et al. Heterogeneous cortical atrophy patterns in $\mathrm{MCl}$ not captured by conventional diagnostic criteria. Neurology 2016;87:2108-16.

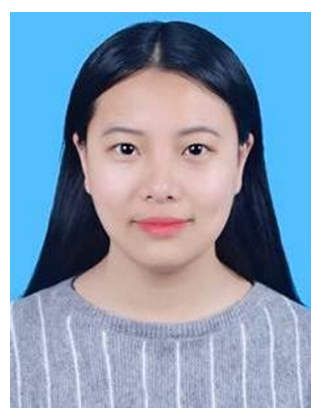

Huanqing Yang graduated from Qiqihar Medical University in 2010 with a bachelor's degree of psychiatry. Now, she is studying for the postgraduate degree in the Shanghai Mental Health Center, Shanghai Jiao Tong University School of Medicine. Her main research areas are the clinical characteristics and the pathogenesis of neurocognitive disorder. 